\title{
Chalcogenide microresonators tailored to distinct morphologies by the shaping of glasses on silica tapers
}

\author{
Ozan Aktaş \\ UNAM-National Nanotechnology Research Center, Bilkent University, Ankara, Turkey (aktaso@bilkent.edu.tr)
}

Received 2 December 2016; revised 10 January 2017; accepted 30 January 2017; posted 1 February 2017 (Doc. ID 282027);

published 17 February 2017

Production of chalcogenide $\left(\mathrm{As}_{2} \mathrm{Se}_{3}\right)$ microresonators in sphere, loop, and bottle morphologies by the shaping of glasses at appropriate temperatures between cleaved silica tapers is reported. The quality factors exceed $Q_{S}=6.2 \times$ $10^{5}, Q_{B}=6.7 \times 10^{5}$, and $Q_{L}=1.6 \times 10^{4}$ for the sphere, bottle, and loop microresonators, respectively. All-optical thermally assisted tuning with a rate of $0.61 \mathrm{~nm} / \mathrm{mW}$ is demonstrated for a bottle microcavity pumped via a silica taper at a wavelength of $670 \mathrm{~nm}$. This technique enables practical and robust in situ production of chalcogenide microresonators thermally spliced to silica fibers in several morphologies with a wide tuning range of size. () 2017 Optical Society of America

OCIS codes: (230.5750) Resonators; (230.3990) Micro-optical devices; (160.2750) Glass and other amorphous materials.

https://doi.org/10.1364/OL.42.000907

Ancient amusement of watching light as it scatters and disperses while propagating through macroscopic glass objects turned into a scientific curiosity when it was discovered that microscopic glass cavities can spatially and temporally confine light at certain wavelengths via total internal reflection on their convex boundary [1]. The phenomenon highly depends on morphological properties of the microcavities. The shaping of glasses at microscale dimensions has been a matter of utmost importance for technological advancement. Eventually, control over morphology has paved the way for optical microresonators in various distinct shapes such as spheres, bottles, cylinders, loops, toroids, disks, and rings [2-5]. Every different resonator shape has its own pros and cons regarding the easiness of production, the highest achievable quality factor, the integrability, and the tunability.

Single discrete optical microresonators have been increasingly demanded for their exploitation in a plethora of applications such as biological sensing [6], optomechanics [7], cavity quantum electrodynamics [8], lasers [9], and all-optical signal processing [10]. Consequently, developing practical and low-cost techniques to produce high-quality-factor glass microresonators in application-specific shapes with a robust way of integration and the capability of sensitive tuning is crucial for photonic applications.

Chalcogenide glasses (ChGs) are currently attracting strong interest as promising materials for active and passive microresonators [11] because of their high refractive index $(n=$ 2.2-3.4), wide transparency in the mid-IR region, high optical nonlinearity, low two-photon absorption, low-temperature processing, and ability to be doped with rare earth elements [12]. Quality factors $Q>10^{7}$ can be achieved with $\mathrm{As}_{2} \mathrm{Se}_{3}$ microcavity resonators [13]. Most of the research on ChGs is dedicated to nonlinear optic applications and mid-IR photonics.

The most easily obtained shape for a glass microresonator is the sphere. Spherical glass microcavities can be produced by reflowing the tip of a tapered glass fiber using resistive heaters [14] or $\mathrm{CO}_{2}$ lasers [15]. Surface tension creates a spherical cavity attached to a fiber stem, which is very convenient for handling. Other techniques compatible with mass production are melting chunks of glass falling freely through a vertical furnace and inducing Plateau-Rayleigh (PR) capillary instability by optical fusing of tapered ChG fibers in mid-air [16] or applying heat to ChG core polymer cladding fibers [17]. These methods depend either on a glass in the form of fiber or tedious selection of a cavity and its attachment to a fiber tip.

The other most closely symmetrical shape is the bottle, as an elongated version of the sphere. The production of bottle cavities generally relies on a two-step heat-and-pull process [18] and soften-and-compress process [19] applied on silica fibers by resistive or laser heating. The only demonstration for a ChG bottle microresonator is the "index bottle" cavity photoinduced in a ChG fiber by exploiting the photosensitivity of $\mathrm{As}_{2} \mathrm{Se}_{3}[20,21]$.

Recently, microfiber loop resonators, for which the problem of optical coupling with a resonator is naturally solved, are drawing significant interest [22], and various applications have been demonstrated [23]. The production of a silica microfiber loop resonator can be easily achieved by creating a self-touching loop pushing the fiber ends together after silica fiber tapering. However, developing $\mathrm{ChG}$ microfiber loop resonators spliced to silica fibers appears to be a challenging task $[24,25]$.

Previously, the direct drawing of photonic microwires from bulk tellurite glasses was demonstrated using sapphire [26] and 
silica fibers [27]. Likewise, a glass wire was directly drawn from a silver phosphate glass and then transferred to a tapered silica fiber. A silver phosphate glass sphere microcavity resonator fixed to the tapered fiber was produced by reflowing the attached glass microwire in a flame [28]. A similar method for the production of $\mathrm{ChG}$ microspheres attached to tapered silica fibers was also reported [29]. Recently, Er:Yb doped phosphate glass bottle microcavities were fabricated on microcapillaries by reflowing some doped glass transferred with a glass rod [30]. These techniques, based on transferring and reflowing of glasses on microfibers, are applicable only for one morphology and, thus, have limited functionality.

In this Letter, a versatile method for production of $\mathrm{ChG}$ microresonators thermally spliced to cleaved silica fiber tapers in sphere, bottle, and loop morphologies was demonstrated. Distinct morphologies were obtained by in situ shaping of ChGs at certain temperatures between cleaved silica tapers, exploiting the surface tension and adhesion forces. Quality factors were obtained as high as $Q_{L}=1.6 \times 10^{4}, Q_{S}=6.2 \times 10^{5}$, and $Q_{B}=6.7 \times 10^{5}$ for fiber loop, sphere, and bottle cavity microresonators, respectively. All-optical thermally assisted tuning of resonance wavelengths was demonstrated with a tuning rate of $0.61 \mathrm{~nm} / \mathrm{mW}$ in a range of $1.2 \mathrm{~nm}$ for an $\mathrm{As}_{2} \mathrm{Se}_{3}$ bottle microcavity pumped via the stem silica taper at a high absorption wavelength $(670 \mathrm{~nm})$ of the glass.

Fabrication of ChG microresonators in different morphologies has three common steps and one last fourth step that is different for each morphology, as shown schematically in Fig. 1. The process starts with silica fiber tapering. The cleaving of a tapered fiber results in cleaved tapers as shown in Fig. 1(a). An electrical heater is used to keep temperature $T$ slightly higher than the melting temperature $T_{m}$ of the $\mathrm{ChG}$ in the following steps. The transfer of ChG to one of the tapers is accomplished in the second step by insertion of the taper into some molten $\mathrm{ChG}$ and its subsequent retraction [see Fig. 1(b)]. In the third step, the ChG attached to one of the tapers can be pulled to the center by the other ChG-free taper, using surface tension and adhesion of the glass. Resulting bottle-like-shaped ChG

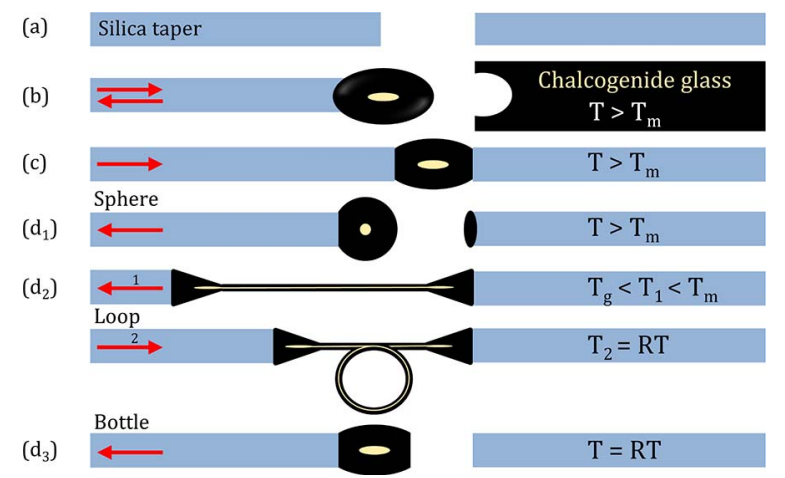

Fig. 1. Production of ChG microresonators in sphere, loop, and bottle morphologies: (a) silica fiber tapering and cleaving, (b) the transfer of some molten ChG to a cleaved silica taper at $T>T_{m}$, i.e., the melting temperature of the $\mathrm{ChG}$, (c) splicing of the transferred $\mathrm{ChG}$ between the tapers at $T>T_{m},\left(d_{1}\right)$ formation of a sphere microcavity after separation at $T>T_{m}$, due to the surface tension, $\left(\mathrm{d}_{2}\right)$ formation of a fiber loop by first drawing a tapered $\mathrm{ChG}$ fiber between the tapers at $T_{g}<T_{1}<T_{m}$, and then bringing the tapers closer at room temperature $T_{2}=R T,\left(\mathrm{~d}_{3}\right)$ formation of a bottle microcavity after separation at $T=R T$. between the cleaved tapers can be seen in Fig. 1(c). At this step, the maximization of transmission of light through the tapers assures the alignment necessary to shape the glass into perfect cylindrical symmetry. At the last step, three different morphologies can be obtained, depending on the temperature $T$. If $T>T_{m}$, the separation of the tapers results in a sphere microcavity on one of the taper tips, due to surface tension [see Fig. $1\left(\mathrm{~d}_{1}\right)$ ]. The bottle-like shape turns into a uniform cylinder after the evaporation of some glass. If, at this point, $T$ is lower than $T_{m}$, but higher than the glass transition temperature $T_{g}$, a tapered ChG fiber can be drawn between the silica tapers. The tapered ChG fiber can be turned into a self-touching loop by bringing the tapers closer to each other at room temperature (RT). As a result, a ChG microfiber loop resonator spliced to silica tapers is obtained [see Fig. $1\left(\mathrm{~d}_{2}\right)$ ]. If $T=R T$, the separation of the tapers results in a bottle microcavity on one of the tapers, preserving the initial shape [see Fig. $\left.1\left(d_{3}\right)\right]$.

At first, $\mathrm{As}_{2} \mathrm{Se}_{3}$ was prepared from high-purity As and Se elements (Sigma Aldrich) using a sealed-ampule melt-quenching technique. A SMF-28 silica fiber was striped over $2 \mathrm{~cm}$ and cleaned with alcohol. Then, the fiber was clamped by two fiber holders fixed on two motorized linear stages (MFA-CC and Newport ESP300 controller), one of which was placed on a three-axis linear stage for 3D manipulation of silica tapers. The silica fiber was tapered in the flame of a hydrogen torch, and then cleaved at the center of the waist with a sharp SiC blade. The cleaved tapers with a tip diameter of $33 \mu \mathrm{m}$ can be seen in Fig. 2(a). For the next step, a small amount of bulk ChG $(<1 \mathrm{mg})$ on a cover slip cantilever was positioned in the space between the cleaved tapers. A custom-made electric heater under ambient air was used to reach temperatures enough to melt $\mathrm{As}_{2} \mathrm{Se}_{3}$ glass $\left(T_{m}=330^{\circ} \mathrm{C}, T_{g}=170^{\circ} \mathrm{C}\right) . \mathrm{An} \mathrm{As}_{2} \mathrm{Se}_{3}$ residue outside the heater was also observed due to the evaporation. A silica taper after its immersion into molten ChG can be seen in Figs. 2(b) and 2(c). Then, the transferred glass was spliced to the silica tapers at $T>T_{m}$ [see Fig. 2(d)]. The separation of the silica tapers at $T>T_{m}$ resulted in a spherical cavity, as shown in Fig. 2(e). The evaporation of the glass at $T>T_{m}$

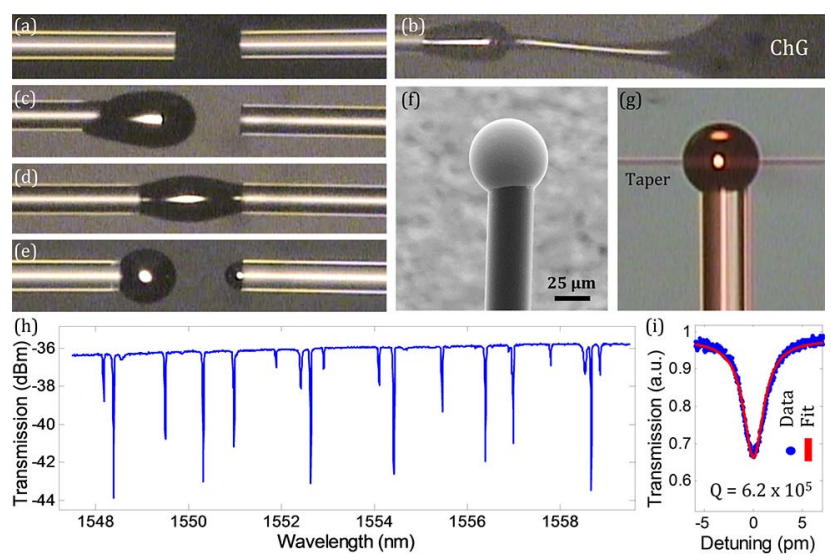

Fig. 2. ChG sphere microcavities: optical micrographs of (a) cleaved silica tapers, (b) a silica taper after its immersion into some molten ChG (see Visualization 1), (c) transfer of the ChG to a silica taper, (d) splicing of the ChG between the tapers, and (e) a sphere microcavity with a diameter of $56 \mu \mathrm{m}$ (see Visualization 2). (f) SEM and (g) optical micrographs of the $\mathrm{As}_{2} \mathrm{Se}_{3}$ sphere microcavity. (h) Transmission spectrum. (i) Lorentzian fit to a resonance mode at $1549.5 \mathrm{~nm}$. 
can be stopped when the desired volume for the cavity is reached. SEM micrograph of the $\mathrm{As}_{2} \mathrm{Se}_{3}$ sphere cavity can be seen in Fig. 2(f). Later, the sphere microcavity was transferred to a three-axis closed loop piezo stage (Thorlabs Max311D/M) in vertical orientation [see Fig. 2(g)], and a new tapered silica fiber was prepared for optical characterizations. Obtained quality factors are over $Q_{S}=6.2 \times 10^{5}$. The transmission spectrum of the sphere microcavity and a Lorentzian fit to a resonance mode at $1549.5 \mathrm{~nm}$ wavelength are shown in Figs. 2(h) and 2(i). The free spectral range (FSR) is $6.03 \mathrm{~nm}$.

For microfiber loop resonators, the transfer of $\mathrm{ChG}$ to the tip of a silica taper with a tip diameter of $35.9 \mu \mathrm{m}$ was obtained in a similar way. At the splicing step, the evaporation of some $\mathrm{ChG}$ is required to form a uniform cylinder between the silica taper tips, as seen in Fig. 3(a); otherwise, tapering will not start at the center, but at one of the weakest splicing points. A tapered ChG fiber was formed by drawing the glass bilaterally with the silica tapers [see Fig. 3(b)] at $T_{m}>T>T_{g}$. The tapering process was also monitored by measuring the transmission of an optical input of $1 \mathrm{~mW}$ at $1550 \mathrm{~nm}$ wavelength. The ChG fiber tapering was stopped when a single-mode regime was reached at a submicron waist diameter. The tapering length is $1.67 \mathrm{~mm}$. The total insertion loss is $9.8 \mathrm{~dB}$, which is the sum of $0.2 \mathrm{~dB}$ silica fiber tapering loss, $0.5 \mathrm{~dB}$ Fresnel loss for each ChG/silica interface, 5.6 dB ChG fiber tapering loss, and $3 \mathrm{~dB}$ loss due to cleaving and mode mismatch. The ChG fiber tapering loss is increased by non-adiabaticity, surface roughness, and Rayleigh scattering. Losses can be mitigated using inert gas atmosphere and angled cleaved fiber tapers. More information about the tapering setup including the electrical heater can be found in a recent study dedicated to ChG fiber tapering [31]. Bringing the tapers closer resulted in a self-touching fiber loop held by van der Waals or electrostatic forces. The loop diameter is tunable via control of the separation length. Optical and SEM micrographs of the ChG microfiber loop resonator are shown in Figs. 3(c) and 3(d). The diameter of the loop and the tapered ChG fiber at the self-coupling point are $178.2 \mu \mathrm{m}$

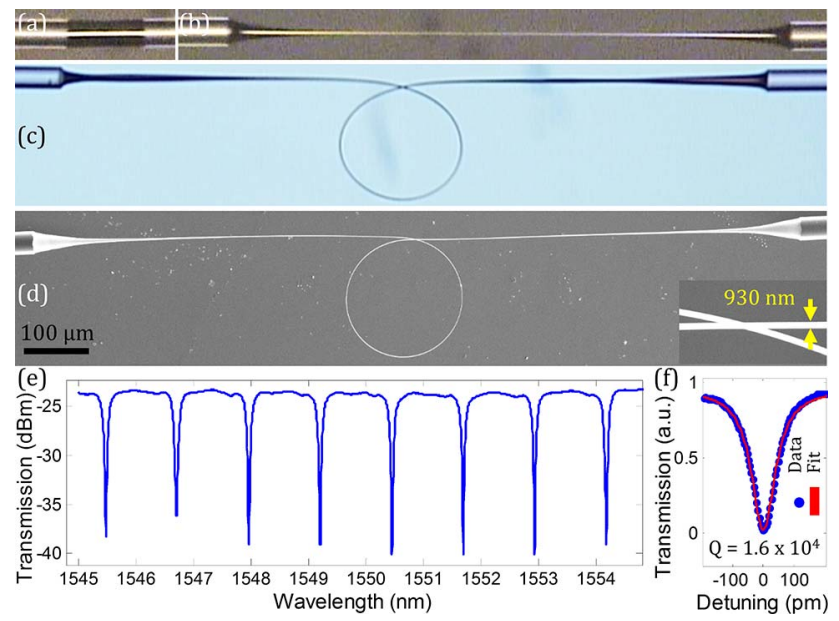

Fig. 3. ChG microfiber loop resonators: optical micrographs of (a) a ChG cylinder between the silica tapers after evaporation of some material, (b) a tapered ChG fiber drawn between the silica tapers (see Visualization 3), (c) a fiber loop obtained by bringing the tapers closer (see Visualization 4). (d) SEM micrograph of the $\mathrm{As}_{2} \mathrm{Se}_{3}$ fiber loop resonator. The inlet shows the self-coupling region. (e) Transmission spectrum. (f) Lorentzian fit to a resonance mode at $1552.9 \mathrm{~nm}$. and $930 \mathrm{~nm}$, respectively. The transmission spectrum of $\mathrm{ChG}$ microfiber loop resonator was observed to be enhanced after annealing at $T=T_{g}$, similar to the enhancement of merging silica microfiber loops with $\mathrm{CO}_{2}$ lasers [32]. The transmission spectrum of the loop resonator and a Lorentzian fit to a resonance mode at $1552.9 \mathrm{~nm}$ wavelength can be seen in Figs. 3(e) and 3(f). Quality factors and coupling strengths are over $Q_{L}=1.6 \times 10^{4}$ and $16.4 \mathrm{~dB}$, respectively. The FSR is $1.24 \pm$ $0.01 \mathrm{~nm}$ in a $10 \mathrm{~nm}$ range.

Another single-mode fiber (Thorlabs S630, a core/cladding diameter of $3.5 / 125 \mu \mathrm{m}$ ) was tapered and cleaved for a $\mathrm{ChG}$ bottle cavity. Some $\mathrm{As}_{2} \mathrm{Se}_{3}$ glass was transferred to one of the tapers and spliced on both sides to the tapers, as shown in Fig. 4(a). The shape and size of a bottle cavity can be tailored by changing the neck-to-neck distance, the neck diameter, and the surface curvature. The separation of the tapers at $T=R T$ resulted in a bottle cavity with a $32.5 \mu \mathrm{m}$ neck and a $54.7 \mu \mathrm{m}$ equator diameter, as shown in Fig. 4(b). SEM and optical micrographs of the bottle cavity can be seen in Figs. 4 (c) and 4(d). The transmission spectrum and the Lorentzian fit to a resonance mode at $1550.44 \mathrm{~nm}$ wavelength are shown in Figs. 4(e) and 4(f). The $Q$-factors exceed $Q_{B}=6.7 \times 10^{5}$, and the FSR is $3.03 \mathrm{~nm}$.

$\mathrm{As}_{2} \mathrm{Se}_{3}$ has a very high absorption at wavelengths below $815 \mathrm{~nm}$, which is convenient for thermo-optical tuning. The temperature control of a refractive index and thermal expansion is more practical than mechanical strain tuning. As an application, the $\mathrm{As}_{2} \mathrm{Se}_{3}$ bottle microcavity was pumped via the stem silica taper at $670 \mathrm{~nm}$ wavelength. An experimental setup consists of a tunable CW IR laser (Santec TLS-510), a CW visible laser (Toptica DL Pro), a polarization controller, an optical power meter (Newport 1935C with 918D-IR-0D3R and 918D-SL-OD3), an attenuator, and a collimator, as shown in Fig. 5(a). The output of the pump laser was free-space-coupled through the attenuator and the collimator lens into the fiber, which is spliced to the bottle cavity on the other tapered end. Before the attachment of the bottle cavity, the pump power was calibrated from the tip of the stem taper at different power levels set by the attenuator. The ChG bottle cavity was positioned close to the tapered silica fiber, assuring alignment with the equator, as shown in Fig. 5(b). The wavelength of the IR laser source was scanned continuously for a $3 \mathrm{~nm}$ range with a scan rate of $3 \mathrm{~nm} / \mathrm{s}$. From $0 \mathrm{~mW}$ to $2 \mathrm{~mW}$ pump powers, the wavelength shifts in the spectrum were recorded, as shown in

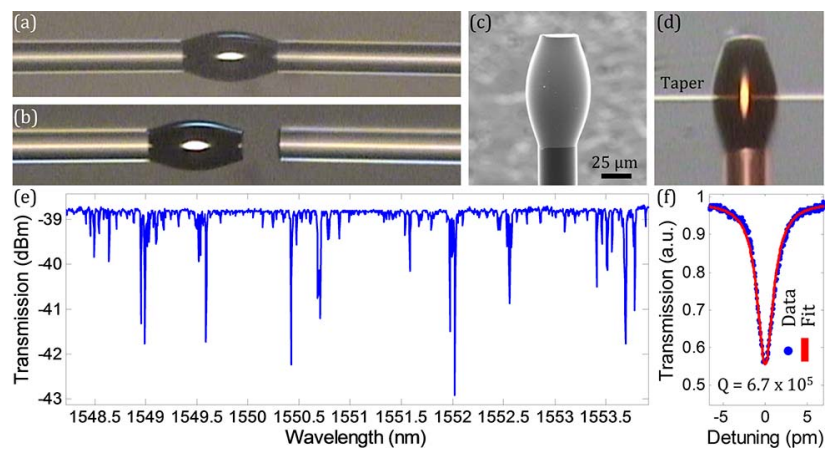

Fig. 4. ChG bottle microcavities: optical micrographs of (a) a spliced ChG between the tapers and (b) a bottle cavity after separation of the tapers at $T=R T$ (see Visualization 5). (c) SEM and (d) optical micrographs of the $\mathrm{As}_{2} \mathrm{Se}_{3}$ bottle microcavity. (e) Transmission spectrum. (f) Lorentzian fit to a resonance mode at $1550.44 \mathrm{~nm}$. 


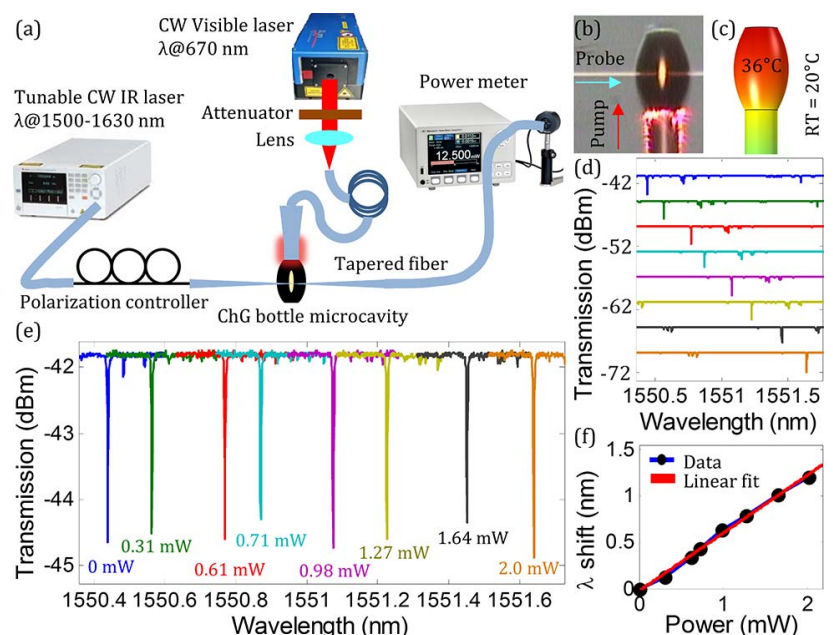

Fig. 5. All-optical thermally assisted tuning of ChG bottle microcavities: (a) experimental setup, (b) optical micrograph of an $\mathrm{As}_{2} \mathrm{Se}_{3}$ bottle cavity microresonator, (c) simulation of pump heating, (d) wavelength shifts of the spectrum at different pump powers, (e) wavelength shifts of a resonance mode at different pump powers, and (f) pump power versus wavelength shift of the resonance mode.

Fig. 5(d) with $4 \mathrm{dBm}$ offsets. The wavelength shift of the resonance mode that is initially at $1550.44 \mathrm{~nm}$ can be seen in Fig. 5(e). A pump-power-limited tuning range of $1.2 \mathrm{~nm}$ was obtained. A tuning rate of $0.61 \mathrm{~nm} / \mathrm{mW}$ was calculated, as shown in Fig. 5(f). Using the thermo-optic and the thermal expansion coefficients of $\mathrm{As}_{2} \mathrm{Se}_{3}$ (AMTIR-2), it was calculated that this rate corresponds to a $15.48^{\circ} \mathrm{C}$ increase in the temperature of the ChG bottle microcavity pumped at $1 \mathrm{~mW}$, which was verified by COMSOL simulations based on the BeerLambert law of laser heating [see Fig. 5(c)]. Pumping via stem fiber can reduce the power requirement to $1 \mathrm{~mW}$ level, as compared to that of free-space coupling.

In conclusion, a practical method for production of $\mathrm{ChG}$ microresonators in several distinct morphologies is demonstrated by in situ shaping of glasses thermally spliced to cleaved silica fiber tapers. Obtained $Q$-factors are as high as $Q_{L}=$ $1.6 \times 10^{4}$ for the microfiber loop, $Q_{S}=6.2 \times 10^{5}$ for the microsphere cavity, and $Q_{B}=6.7 \times 10^{5}$ for the microbottle cavity resonators. Although the sphere and the bottle microcavities have slightly higher quality factors than those of the $\mathrm{As}_{2} \mathrm{Se}_{3}$ microcavities previously obtained by inducing PR instability, further improvements can be achieved using inert gas atmosphere and purified $\mathrm{ChGs}$ for the production. The highest $Q$-factor reported so far for $\mathrm{As}_{2} \mathrm{Se}_{3}$ is $2.3 \times 10^{6}$. This technique can also be applied to other common ChGs, including $\mathrm{As}_{2} \mathrm{Se}_{3}$, As-Se-S, and Ge-As-Se-Te in very low starting quantities, without the need for a ChG in the form of fiber. The ChG microresonators thermally spliced to cleaved silica fiber tapers, eliminate post-process splicing or attachment of cavities with glues. Other types of resonators such as cylinders, oblate spheroids, and all-fiber F-P cavities are also obtainable with this method. As an application, all-optical thermally assisted tuning with a rate of $0.61 \mathrm{~nm} / \mathrm{mW}$ was demonstrated for an $\mathrm{As}_{2} \mathrm{Se}_{3}$ bottle microcavity resonator pumped via the stem fiber at $670 \mathrm{~nm}$ wavelength. ChG microresonators can be exploited for nonlinear optical applications and mid-IR photonics.
With the capability of thermo-optical tuning, these resonators can serve as tunable filters or tunable feedback elements for quantum cascade lasers. Pumping via a stem fiber was also reported for microcavity lasers [33].

Funding. European Research Council (ERC) (307357).

Acknowledgment. The author thanks Prof. Mehmet Bayındır for his valuable guidance and inspiration, Dr. Erol Özgür for his help in taking SEM micrographs, and Dr. Seymur Cahangirov for his advice.

\section{REFERENCES}

1. R. D. Richtmyer, J. Appl. Phys. 10, 391 (1939).

2. K. J. Vahala, Nature 424, 839 (2003).

3. J. Ward and O. Benson, Laser Photon. Rev. 5, 553 (2011).

4. J. Wang, T. Zhan, G. Huang, P. K. Chu, and Y. Mei, Laser Photon. Rev. 8, 521 (2014).

5. L. Gai, J. Li, and Y. Zhao, Opt. Laser Technol. 89, 126 (2017).

6. F. Vollmer, S. Arnold, and D. Keng, Proc. Natl. Acad. Sci. USA 105, 20701 (2008).

7. Y. L. Li, J. Millen, and P. F. Baker, Opt. Express 24, 1392 (2016).

8. D. O'Shea, C. Junge, J. Volz, and A. Rauschenbeutel, Phys. Rev. Lett. 111, 193601 (2013).

9. G. R. Elliott, G. S. Murugan, J. S. Wilkinson, M. N. Zervas, and D. W. Hewak, Opt. Express 18, 26720 (2010).

10. M. Pöllinger and A. Rauschenbeutel, Opt. Express 18, 17764 (2010).

11. G. R. Elliott, D. W. Hewak, G. S. Murugan, and J. S. Wilkinson, Opt. Express 15, 17542 (2007).

12. B. J. Eggleton, B. Luther-Davies, and K. Richardson, Nat. Photonics 5, 141 (2011).

13. F. Vanier, M. Rochette, N. Godbout, and Y.-A. Peter, Opt. Express 38 4966 (2013).

14. D. H. Broaddus, M. A. Foster, I. H. Agha, J. T. Robinson, M. Lipson, and A. L. Gaeta, Opt. Express 17, 5998 (2009).

15. C. Guo, K. Che, P. Zhang, J. Wu, Y. Huang, H. Xu, and Z. Cai, Opt. Express 23, 32261 (2015).

16. C. Grillet, S. N. Bian, E. C. Magi, and B. J. Eggleton, Appl. Phys. Lett. 92, 171109 (2008).

17. O. Aktas, E. Ozgur, O. Tobail, M. Kanik, E. Huseyinoglu, and M. Bayindir, Adv. Opt. Mater. 2, 618 (2014).

18. M. Pöllinger, D. O'Shea, F. Warken, and A. Rauschenbeutel, Phys. Rev. Lett. 103, 053901 (2009).

19. G. S. Murugan, J. S. Wilkinson, and M. N. Zervas, Opt. Express 17 11916 (2009)

20. F. Luan, E. Magi, T. Gong, I. Kabakova, and B. J. Eggleton, Opt. Lett. 36, 4761 (2011)

21. A. L. Watts, N. Singh, C. G. Poulton, E. C. Magi, I. V. Kabakova, D. D. Hudson, and B. J. Eggleton, J. Opt. Soc. Am. B 30, 3249 (2013).

22. M. Sumetsky, Y. Dulashko, and A. Hale, Opt. Express 12, 3521 (2004).

23. R. Ismaeel, T. Lee, M. Ding, N. G. R. Broderick, and G. Brambilla, Opt. Lett. 37, 5121 (2012).

24. G. Vienne, A. Coillet, P. Grelu, M. El Amraoui, J.-C. Jules, F. Smektala, and L. Tong, Opt. Express 17, 6224 (2009).

25. Q. Zhang, M. Li, Q. Hao, D. Deng, H. Zhou, H. Zeng, L. Zhan, X. Wu, L. Liu, and L. Xu, Opt. Lett. 35, 3829 (2010).

26. L. Tong, L. Hu, J. Zhang, J. Qu, Q. Yang, J. Lou, Y. Shen, J. He, and Z. Ye, Opt. Express 14, 82 (2006).

27. N.-K. Chen, P.-W. Kuan, J. Zhang, L. Zhang, L. Hu, C. Lin, and L. Tong, Opt. Express 18, 25615 (2010).

28. K. Milenko, I. Konidakis, and S. Pissadakis, Opt. Lett. 41, 2185 (2016)

29. H. Ahmad, I. Aryanfar, K. S. Lim, W. Y. Chong, and S. W. Harun, Quantum Electron. 42, 462 (2012).

30. J. M. Ward, Y. Yang, and S. N. Chormaic, Sci. Rep. 6, 25152 (2016).

31. O. Aktas and M. Bayindir, Appl. Opt. 56, 385 (2017)

32. P. Pal and W. H. Knox, IEEE Photon. Technol. Lett. 21, 766 (2009).

33. A. Watkins, J. Ward, and S. N. Chormaic, Jpn. J. Appl. Phys. 51, 052501 (2012). 